\title{
Asymptotic Expressions for the Bessel Functions and the Fourier-Bessel Expansions.
}

By Dr T. M. MacRobert.

(Read 14th January 1921. Received 4th March 1921.)

\section{Part I.}

Asymptotic Expressions for the Bessel Functions.

From the asymptotic expansion for $K_{n}(z)$ it follows that, if $-\pi<\operatorname{amp} z<\pi$,

$$
\operatorname{Lim}_{z \rightarrow \infty} K_{n}(z) / \sqrt{ }\left\{\left(\frac{\pi}{2 z}\right) e^{-z}\right\}=1 .
$$

This theorem is also true if $\operatorname{amp} z= \pm \pi$; to prove this consider the formula

$$
K_{n}(z)=\sqrt{ }\left(\frac{\pi}{2 z}\right) \frac{1}{\Gamma\left(n+\frac{1}{2}\right)} e^{-z} \int_{0}^{\infty} e^{-\xi} \xi^{n-1}\left(1+\frac{\xi}{2 z}\right)^{n-1} d \xi .
$$

If $R\left(n+\frac{1}{2}\right)>0$, this formula is valid for $z \neq 0,-\pi<$ ump $z<\pi$, since both sides of the equation are holomorphic in this region. Now let $z=x e^{i \theta}$, where $x$ is real and positive, and let the path of integration be deformed into the contour consisting of : (i) the $\xi$-axis from 0 to $2 x-\epsilon$; (ii) a semicircle of centre $2 x$ and radius $\epsilon$ lying above the $\xi$-axis; (iii) the $\xi$-axis from $2 x+\epsilon$ to $\infty$. Then the integral is holomorphic in $z$ at $z=x e^{i \pi}$. If $\theta=-\pi$, the semicircle is taken to lie below the $\xi$-axis. Since $R\left(n+\frac{1}{2}\right)>0$, the integral round the semi-circle tends to zero with $\epsilon$.

$$
\begin{aligned}
& \text { Hence, if } z=x e^{ \pm i \pi} \text {, } \\
& K_{n}(z)=\sqrt{ }\left(\frac{\pi}{2 z}\right) \frac{1}{\Gamma^{\prime}\left(n+\frac{1}{2}\right)} e^{-z}\left\{I_{1}+e^{\mp i \pi\left(n-\frac{1}{2}\right)} I_{2}\right\}, \\
& \text { where } \quad I_{1}=\int_{0}^{2 x} e^{-\xi} \xi^{n-\frac{1}{2}}\left(1-\frac{\cdot \xi}{2 x}\right)^{n-1} d \xi \text {, } \\
& \text { and } \quad I_{2}=\int_{2 x}^{\infty} e^{-\xi} \xi^{n-\frac{1}{2}}\left(\frac{\xi}{2 x}-1\right)^{n-\frac{1}{2}} d \xi \text {. }
\end{aligned}
$$


14

Now let $M$ be a large positive quantity less than $x$; then

$$
\begin{gathered}
I_{1}=\int_{0}^{M} e^{-\xi} \xi^{n-\frac{1}{2}}\left(1-\frac{\xi}{2 x}\right)^{n-1} d \xi+V, \\
V=\int_{M}^{2 x} e^{-\xi} \xi^{n-1}\left(1-\frac{\xi}{2 x}\right)^{n-1} d \xi .
\end{gathered}
$$

Then, if $R(n)=\alpha$,

$$
|V| \leqq \int_{\mu}^{2 x} e^{-\xi} \xi^{a-\frac{1}{2}}\left(1-\frac{\xi}{2 x}\right)^{a-1} d \xi
$$

Two cases have to be considered; namely,

$$
\alpha \geqq \frac{1}{2} \text { and }-\frac{1}{2}<\alpha<\frac{1}{2} \text {. }
$$

Case I. Let $a \geqq \frac{1}{2}$; then, since $\left(1-\frac{\xi}{2 x}\right)<1$,

$$
|V| \leqq \int_{M}^{2 x} e^{-\xi} \xi^{a-\frac{1}{2}} d \xi
$$

Case II. Let $-\frac{1}{2}<\alpha<\frac{1}{2}$; then

$$
\begin{aligned}
|V| \leqq \int_{M}^{x} e^{-\xi} \xi^{a-\frac{1}{2}}\left(1-\frac{\xi}{2 x}\right)^{a-\frac{1}{2}} d \xi & \\
& \quad+\int_{x}^{2 x} e^{-\xi} \xi^{a-\frac{1}{2}}\left(1-\frac{\xi}{2 x}\right)^{a-\frac{1}{2}} d \xi .
\end{aligned}
$$

Accordingly, by the First Mean Value Theorem

$$
\begin{aligned}
|V| \leqq \lambda \int_{M}^{x} e^{-\xi} \xi^{a-\frac{1}{2}} d \xi & \\
& +e^{-(1+\theta) x}(1+\theta)^{a-\frac{1}{2}} x^{a-\frac{1}{2}} \int_{x}^{2 x}\left(1-\frac{\xi}{2 x}\right)^{a-\frac{1}{2}} d \xi,
\end{aligned}
$$

where $1<\lambda<2^{\frac{1}{2-a}}$ and $0<\theta<1$.

Thus in both cases

$$
\begin{gathered}
\operatorname{Lim}_{x \rightarrow \infty}|V| \leqq \lambda \int_{M}^{\infty} e^{-\xi} \xi^{a-\frac{1}{1}} d \xi . \\
\text { But } \operatorname{Lim}_{x \rightarrow \infty} \int_{0}^{M} e^{-\xi} \xi^{n-\frac{1}{2}}\left(1-\frac{\xi}{2 x}\right)^{n-\frac{1}{2}} d \xi=\int_{0}^{M} e^{-\xi} \xi^{n-\frac{1}{2}} d \xi .
\end{gathered}
$$

https://doi.org/10.1017/S0013091500035744 Published online by Cambridge University Press 
Hence, by making $M$ tend to infinity, it follows that

$$
\operatorname{Lim}_{x \rightarrow \infty} I_{1}=\int_{0}^{\infty} e^{-\xi} \xi^{n-1} d \xi=\Gamma\left(n+\frac{1}{2}\right) .
$$

In the next place

$$
\left|I_{2}\right| \leqq \int_{2 x}^{\infty} e^{-\xi} \xi^{a-1}\left(\frac{\xi}{2 x}-1\right)^{a-\frac{1}{3}} d \xi .
$$

Here put $\xi=2 x(1+\eta)$; then

$$
\begin{aligned}
\left|I_{z}\right| & \leqq e^{-2 x}(2 x)^{a+1} \int_{0}^{\infty} e^{-2 x \eta}(1+\eta)^{a-\frac{1}{2}} \eta^{a-1} d \eta \\
& <0^{-2 x}(2 x)^{a+\frac{1}{z}} \int_{0}^{\infty} e^{-\eta}(1+\eta)^{a-\frac{1}{2}} \eta^{\alpha-\frac{1}{2}} d \eta .
\end{aligned}
$$

Hence $\operatorname{Lim}_{x \rightarrow \infty} I_{2}=0$.

Accordingly, if amp $z= \pm \pi$,

$$
\operatorname{Lim}_{z \rightarrow \infty} K_{n}(z) /\left\{\sqrt{ }\left(\frac{\pi}{2 z}\right) e^{-s}\right\}=1 .
$$

Since $K_{-n}(z)=K_{n}(z)$, this is true for all values of $n$.

The corresponding theorems for the other Bessel Functions can be deduced from this. They are

$$
\lim _{z \rightarrow \infty} G_{n}(z) /\left\{\sqrt{ }\left(\frac{\pi}{2 z}\right) e^{-\frac{1}{2} n \pi i+i(z+\pi / 4)}\right\}=1,
$$

where $-\pi / 2 \leqq$ amp $z \leqq 3 \pi / 2$;

$$
\operatorname{Lim}_{z \rightarrow \infty} J_{n}(z) /\left\{\sqrt{ }\left(\frac{2}{\pi z}\right) \cos (z-\pi / 4-n \pi / 2)\right\}=1,
$$

where $-\pi / 2 \leqq \operatorname{amp} z \leqq \pi / 2$;

$$
\operatorname{Lim}_{z \rightarrow \infty} J_{n}(z) /\left\{i e^{i n \pi} \sqrt{ }\left(\frac{2}{\pi z}\right) \cos (z+\pi / 4+n \pi / 2)\right\}=1,
$$

where $\pi / 2 \leqq \operatorname{amp} z \leqq 3 \pi / 2$;

$$
\operatorname{Lim}_{z \rightarrow \infty} I_{n}(z) /\left\{\frac{1}{\sqrt{ }(2 \pi z)} e^{z}+e^{-i\left(n+\frac{1}{2}\right) \pi} \frac{1}{\sqrt{ }(2 \pi z)} e^{-z}\right\}=1,
$$

where $-\pi \leqq \operatorname{amp} z \leqq 0$; 


$$
\operatorname{Lim}_{z \rightarrow \infty} I_{n}(z) /\left\{\frac{1}{\sqrt{(2 \pi z)}} e^{z}+e^{i(n+3) \pi} \frac{1}{\sqrt{ }(2 \pi z)} e^{-z}\right\}=1 \text {, }
$$

where $0 \leqq \operatorname{amp} z \leqq \pi$.

Part II.

The Fourier-Bessel Expansions.

These theorems make it possible to establish the validity of the Fourier-Bessel Expansions by means of Coutour Integration. For simplicity, the expansion

$$
f(r)=\sum_{s=1}^{\infty} A_{s} J_{0}\left(\lambda_{s} r\right)
$$

where $\lambda_{1}, \lambda_{2}, \ldots$ are the positive zeros of $J_{0}(x)$ will first be considered.

Here

$$
A_{s}=2 \frac{\int_{0}^{1} x f(x) J_{0}\left(\lambda_{1} x\right) d x}{\left\{J_{0}^{\prime}\left(\lambda_{s}\right)\right\}^{2}},
$$

so that if $S_{n}$ is the sum of the first $n$ terms of the series on the right-hand side of (1),

$$
S_{n}=2 \int_{0}^{1} x f(x) \sum_{s=1}^{n} \frac{J_{0}\left(\lambda_{t} x\right) J_{0}\left(\lambda_{t} r\right)}{\left\{J_{0}^{\prime}\left(\lambda_{s}\right)\right\}^{2}} d x
$$

Let the integral

$$
\int \frac{\zeta G_{n}(\zeta) J_{0}(\zeta x) J_{0}(\zeta r)}{J_{0}(\zeta)} d \zeta
$$

be taken round the contour consisting of the $\xi$-axis from $-M$ to $M$, indented at $\zeta=0$ and at the zeros of $J_{0}(\zeta)$, and the lines $\xi=M, \eta=N$, and $\xi=-M$, where $M$ and $N$ are positive and $M$ is chosen to lie between the zeros $\lambda_{\nu}$ and $\lambda_{\nu+1}$. The integrand is holomorphic within the contour, so that the value of the integral is zero.

The integral round the small semi-circle at $\zeta=0$ tends to zero with the radius. Also, since

$$
G_{0}(\zeta) J_{0}^{\prime}(\zeta)-J_{0}(\zeta) G_{0}^{\prime}(\zeta)=1 / \zeta
$$


it follows that $\lambda_{s} G_{0}\left(\lambda_{s}\right)=1 / J_{0}^{\prime}\left(\lambda_{s}\right)$; hence the sum of the integrals round the small semi-circles at the zeros of $J_{0}(\zeta)$ tends to

$$
-2 \pi i \sum_{s=1}^{\nu} \frac{J_{0}\left(\lambda_{s} x\right) J_{0}\left(\lambda_{s} r\right)}{\left\{J_{0}^{\prime}\left(\lambda_{s}\right)\right\}^{2}}
$$

as the radii tend to zero.

Again, along the $\xi$-axis the integrand is uniform and odd apart from the term in $G_{0}(\zeta)$ which involves $\log \zeta$; this latter-term gives rise to an integral

$$
i \pi \int_{0}^{M} \xi J_{0}(\xi x) J_{0}(\xi r) d \xi,
$$

while the remaining integrals from $-M$ to 0 and 0 to $M$ cancel each other.

But

$$
\begin{aligned}
\int_{0}^{M} \xi & J_{0}(\xi x) J_{0}(\xi r) d \xi \\
& =\frac{M}{x^{2}-r^{2}}\left\{r J_{0}(M x) J_{0}^{\prime}(M r)-x J_{0}(M r) J_{0}^{\prime}(M x)\right\} \\
& =\frac{M}{x^{2}-r^{2}}\left\{-r J_{0}(M x) J_{1}(M r)+x J_{0}(M r) J_{1}(M x)\right\} .
\end{aligned}
$$

In the right-hand side of this equation replace the Pesse] Functions by their asymptotic expansions; then

$$
\begin{aligned}
\int_{0}^{M} \xi J_{0}(\xi x) J_{0}(\xi r) d \xi \\
=\frac{2}{\pi \sqrt{(x r)}} \frac{1}{x^{2}-r^{2}}\left\{\begin{array}{l}
-x \cos (M r-\pi / 4) \cos (M x+\pi / 4) \\
+r \cos (M r+\pi / 4) \cos (M x-\pi / 4)
\end{array}\right\}+\frac{P}{M} \\
=\frac{1}{\pi \sqrt{ }(x r)}\left\{\frac{\sin \{M(x-r)\}}{x-r}-\frac{\cos \{M(x+r)\}}{x+r}\right\}+\frac{P}{M},
\end{aligned}
$$

where $P$ is finite for all values of $M$.

Now let $N$ tend to infinity; then, if $|x+r|<2$, the integral along $\eta=N$ tends to zero. Also, if the Bessel Functions in the integrals along $\xi= \pm M$ be replaced by their asymptotic expansions, these integrals have the values $I_{1}+Q / M$ and $I_{2}+R / M$, where $Q$ and $R$ are finite ${ }^{*}$ and

- This will be clear if $N$ and $M$ tend to infinity together in such a way that the line joining the origin to the point $M+i N$ makes a tinite angle with the imaginary axis ; for instance, if $M I=N$. 


$$
\begin{aligned}
& I_{1}=-\frac{1}{\sqrt{(x r)}} \\
& \int_{0}^{\infty} \frac{e^{i(M+i \eta-\pi / 4)} \cos \{x(M+i \eta)-\pi / 4\} \cos \{r(M+i \eta)-\pi / 4\}}{\cos (M+i \eta-\pi / 4)} d \eta, \\
& I_{2}=\frac{1}{\sqrt{(x r)}} \\
& \int_{0}^{\infty} \frac{e^{i(-M+i \eta+\pi / 4)} \cos \{x(-M+i \eta)+\pi / 4\} \cos \{r(-M+i \eta)+\pi / 4\}}{\cos (-M+i \eta+\pi / 4)} d \eta .
\end{aligned}
$$

Again

$$
\begin{gathered}
\left|\frac{e^{i( \pm M+i \eta \mp \pi / 4)}}{\cos ( \pm M+i \eta \mp \pi / 4}\right|=\left|2 \frac{e^{-2 \eta}}{1+e^{ \pm 2 i(M-\pi / 4)-2 \eta}}\right| \\
\leqq 2 K e^{-2 \eta}
\end{gathered}
$$

where $K$ is a finite positive constant.

But

$$
\begin{aligned}
& I_{1}=-\frac{1}{2 \sqrt{(x r)}} \\
& \int_{0}^{\infty} \frac{e^{i(M+i \eta-\pi / 4)}[\sin \{(x+r)(M+i \eta)\}+\cos \{(x-r)(M+i \eta)\}]}{\cos (M+i \eta-\pi / 4)} d \eta \\
& =\frac{1}{2 \sqrt{(x r)}}\left[\sin \{(x+r) M\} \times V_{1}+\cos \{(x+r) M\} \times V_{2}\right. \\
& \left.\quad+\cos \{(x-r) M\} \times V_{3}+\sin \{(x-r) M\} \times V_{4}\right],
\end{aligned}
$$

where

$$
\begin{aligned}
\left|V_{1}\right| & \leqq 2 K \int_{0}^{\infty} e^{-2 \eta} \cosh \{(x+r) \eta\} d \eta \\
& \leqq K\left(\frac{1}{2+x+r}+\frac{1}{2-x-r}\right),
\end{aligned}
$$

provided that $|x+r|<2$, and similarly for $V_{2}, V_{3}$, and $V_{1} . \quad I_{2}$ can also be expressed as the sum of four similar expressions.

Accordingly, if $0 \leqq r<1$, and since $0 \leqq x \leqq 1$, 


$$
\begin{gathered}
\sum_{s=1}^{\nu} \frac{J_{0}\left(\lambda_{t} x\right) J_{0}\left(\lambda_{s} r\right)}{\left\{J_{0}\left(\lambda_{t}\right)\right\}^{2}}=\frac{1}{2 \pi \sqrt{ }(x r)}\left\{\frac{\sin \{M(x-r)\}}{x-r}-\frac{\cos \{M(x+r)\}}{x+r}\right\} \\
+\frac{P}{2 M}+\sum_{s=1}^{16} \sin \{(x \pm r) M\} W_{s}+\frac{Q^{\prime}}{M}+\frac{R^{\prime}}{M}
\end{gathered}
$$

where $W_{,}, Q^{\prime}$ and $R^{\prime}$ are finite.

Now multiply this equation by $2 x f(x)$, integrate from 0 to 1 , and let $v$ tend to infinity: then

$$
\begin{aligned}
\operatorname{Lim}_{\nu \rightarrow \infty} 2 \int_{0}^{1} x f(x) & \sum_{s=1}^{\nu} \frac{J_{0}\left(\lambda_{s} x\right) J_{0}\left(\lambda_{t} r\right)}{\left\{J_{0}^{\prime}\left(\lambda_{s}\right)\right\}^{2}} \\
& =\frac{1}{\pi} \operatorname{Lim}_{M \rightarrow \infty} \int_{0}^{1} x f(x) \frac{1}{\sqrt{ }(x r)} \frac{\sin \{M(x-r)\}}{x-r} d x \\
& -\frac{1}{\pi} \operatorname{Lim}_{M \rightarrow \infty} \int_{0}^{1} x f(x) \frac{1}{\sqrt{ }(x r)} \frac{\cos \{M(x+r)\}}{x+r} d x \\
& +\sum_{r=1}^{16} \operatorname{Lim}_{M \rightarrow \infty} \int_{0}^{1} x f(x) W \cdot \sin \{(x \pm r) M\} d x .
\end{aligned}
$$

By the theory of Dirichlet Integrals,

$$
\operatorname{Lim}_{M \rightarrow \infty} \int_{a}^{b} \phi(x) \sin _{\cos }(M x) d x=0
$$

provided that, for $a \leqq x \leqq b, \phi(x)$ is nnite and continuous, except for a finite number of finite discontinuities, and has only a finite number of maxima and minima; while, subject to the same conditions,

$$
\operatorname{Lim}_{M \rightarrow \infty} \int_{a}^{b} \phi(x) \frac{\sin \{M(x-r)\}}{x-r} d x=\frac{\pi}{2}\{\phi(r+0)+\phi(r-0)\}
$$

for $a<r<b$.

Accordingly, if $f(x)$ satisfies these conditions for $0 \leqq x \leqq 1$,

$$
\sum_{i=1}^{\infty} A_{s} J_{0}\left(\lambda_{s} r\right)=\frac{1}{2}\{f(r+0)+f(r-0)\},
$$

provided that $0<r<1$.

When $r=1, J_{0}(\lambda, r)=0$, and therefore the sum of the series is zero. 
When $r=0$, it follows from the theory of Dirichlet Integrals that the sum is $\frac{1}{2} f(+0)$.

Similarly for the series $\Sigma A_{a} J_{n}\left(\lambda_{a} r\right)$, where $\lambda_{d}$ is a positive zero of $J_{n}(x)$, consider the integral of $\zeta G_{n}(\zeta) J_{n}(\zeta x) J_{n}(\zeta r) / J_{n}(\zeta)^{*}$ and when $\lambda_{a}$ is a positive zero of $d x J_{n}^{\prime}(x)+B J_{n}(x)$ employ the integral of

$$
\frac{\zeta\left\{A \zeta G_{n}^{\prime}(\zeta)+B G_{n}(\zeta)\right\} J_{n}(\zeta x) J_{n}(\zeta r)}{A \zeta J_{n}^{\prime}(\zeta)+B J_{n}(\zeta)}
$$

In the former case

$$
A_{s}=2 \int_{0}^{1} x f(x) J_{n}\left(\lambda_{s} x\right) d x /\left\{J_{n}^{\prime}\left(\lambda_{2}\right)\right\}^{2}
$$

and in the latter case

$$
\Lambda_{1}=\frac{A^{2} \lambda_{1}^{2}}{\left\{B^{2}+A^{2}\left(\lambda_{1}^{2}-n^{2}\right)\right\} J_{n}^{2}\left(\lambda_{t}\right)} \int_{0}^{1} x f(x) J_{n}\left(\lambda_{b} x\right) d x .
$$

If in (2) $r, x$, and $\lambda$ are replaced by $r / a, x / a$, and $a \lambda$, and $\phi(r)$ is written in place of $f(r / a)$, then the equation may be written

$$
\sum_{i=1}^{\infty} J_{0}\left(\lambda_{s} r\right) \frac{2 \int_{0}^{a} x \phi(x) J_{0}(\lambda, x) d x}{\left\{a J_{0}^{\prime}\left(\lambda_{s} a\right)\right\}^{2}}=\frac{1}{2}\{\phi(r+0)+\phi(r-0)\},
$$

provided that $0<r<a$, where $\lambda_{s}$ is a positive zero of $J_{0}(\lambda a)$. These transformations can also be applied to the other expansions.

* When $n$ is not an integer the integra' along the $\xi \cdot B \times$ is becomes

$$
\begin{aligned}
& P \int_{0}^{M} \frac{\xi \pi\left\{J_{-n}(\xi)-e^{-i n \pi} J_{n}(\xi)\right\} J_{n}(\xi x) J_{n}(\xi r)}{2 \sin n \pi J_{n}(\xi)} d \xi \\
- & P \int_{0}^{M} \frac{{ }^{2} \pi\left\{J_{-n}(\xi)-e^{i n \pi} J_{n}(\xi)\right\} J_{n}(\xi x) J_{n}(\xi r)}{2 \sin n \pi J_{n}(\xi)} d \xi \\
= & i \pi \int_{0}^{M} \xi J_{n}(\xi x) J_{n}(\xi r) d \xi .
\end{aligned}
$$

